\title{
Manutenção da qualidade de rosas cortadas cv. Vega em soluções conservantes
}

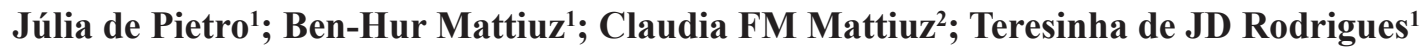 \\ ${ }^{1}$ UNESP-FCAV, Rodov. Prof. Paulo Donato Castellane, s/nº, 14884-900 Jaboticabal-SP; benhur@fcav.unesp.br; ${ }^{2}$ Centro Univ. Moura \\ Lacerda, Unid. II Av. Dr. Oscar de Moura Lacerda 1520, 14076-510 Ribeirão Preto-SP
}

\section{RESUMO}

A rosa é considerada uma das flores de corte mais tradicionais e requisitadas no mercado mundial. Entretanto, após o corte, essas flores tornam-se altamente perecíveis devido à elevada atividade metabólica que conduz aos processos de senescência. Assim, o experimento foi realizado para verificar o efeito do uso de soluções conservantes na fisiologia pós-colheita de rosas vermelhas cortadas cultivar Vega. Utilizou-se o delineamento inteiramente casualizado, em esquema fatorial composto por dois fatores: sete tratamentos pós-colheita e quatro datas de avaliação. As flores permaneceram nas seguintes soluções de manutenção: 1) Água destilada; 2) 8-hidroxiquinolina (200 $\left.\mathrm{mg} \mathrm{L}^{-1}\right)$; 3) 8-hidroxiquinolina (200 $\left.\mathrm{mg} \mathrm{L}^{-1}\right)+$ Sacarose (20 mg L-1); 4) Ácido cítrico $\left.\left(75 \mathrm{mg} \mathrm{L}^{-1}\right) ; 5\right)$ Ácido cítrico $\left(75 \mathrm{mg} \mathrm{L}^{-1}\right)+$ Sacarose (20 mg L-1 $)$; 6) 6-benziladenina (60 $\left.\mathrm{mg} \mathrm{L}^{-1}\right)$; 7) 6 benziladenina (60 $\left.\mathrm{mg} \mathrm{L}^{-1}\right)+$ Sacarose $\left(20 \mathrm{mg} \mathrm{L}^{-1}\right)$. Foram realizadas análises qualitativas e fisiológicas ao longo do período de avaliação. O tratamento com 8-hidroxiquinolina e ácido cítrico revelaram-se mais promissores para manter a qualidade e prolongar a vida de vaso das flores, o que foi evidenciado pela menor perda de massa fresca, atividade respiratória e conteúdo relativo de água; melhor manutenção do conteúdo de carboidratos solúveis e redutores, antocianina e coloração.

Palavras-chave: Rosa sp., pós-colheita, flor de corte, compostos químicos, fitorreguladores.

\section{ABSTRACT}

Keeping quality of cut roses cv. Vega in holding solutions

The rose is considered one of the most traditional and required cut flowers in the world market. However, after cutting, these flowers become highly perishable, because the high metabolic activity leads to senescence processes. The experiment was accomplished to verify the effect of holding solutions on the postharvest physiology of cut red roses, cv. Vega. A randomized design in a factorial scheme composed by two factors was used: seven postharvest treatments and four evaluation dates. The flowers remained in the following holding solutions: 1) Distilled water; 2) 8-hydroxyquinoline citrate (200 mg $\left.\mathrm{L}^{-1}\right)$; 3) 8-hydroxyquinoline citrate $\left(200 \mathrm{mg} \mathrm{L}^{-1}\right)+$ Sucrose $(20 \mathrm{mg}$ $\left.\mathrm{L}^{-1}\right)$; 4) Citric acid (75 $\left.\left.\mathrm{mg} \mathrm{L}^{-1}\right) ; 5\right)$ Citric acid $\left(75 \mathrm{mg} \mathrm{L}^{-1}\right)+$ Sucrose (20 $\left.\mathrm{mg} \mathrm{L}^{-1}\right) ; 6$ ) 6-benzyladenine $\left.\left(60 \mathrm{mg} \mathrm{L}^{-1}\right) ; 7\right)$ 6-benzyladenine (60 $\left.\mathrm{mg} \mathrm{L}^{-1}\right)+$ Sucrose $\left(20 \mathrm{mg} \mathrm{L}^{-1}\right)$. Qualitative and physiologic analyses were carried out during the evaluation period. The treatment with 8-hydroxyquinoline citrate and citric acid were the most promising to keep the quality and extend the vase life of flowers content; better maintenance of soluble and reducing carbohydrates, anthocyanin and color.

Keywords: Rosa sp., postharvest, cut flower, chemicals, phytoregulators.

(Recebido para publicação em 21 de dezembro de 2010; aceito em 30 de janeiro de 2012) (Received on December 21, 2010; accepted on January 30, 2012)

\begin{abstract}
$\mathrm{A}^{\mathrm{s}}$ s flores de corte são importantes produtos da floricultura brasileira, responsáveis por $29 \%$ da área cultivada com flores e plantas no país (Ibraflor, 2011). A produção tem crescido consideravelmente no país, consolidando-se em vários estados. Dentre as flores cortadas, a rosa (Rosa sp) é uma das mais comercializadas no mercado interno (Junqueira \& Peetz, 2007) e exportadas pelo Brasil (Barguil et al., 2010). Takane et al. (2007) estimaram por meio de dados dos principais centros de comercialização atacadista do país, que a produção anual brasileira de rosas seja de 25 milhões de dúzias.
\end{abstract}

Após a colheita, as rosas de corte têm um período de vida útil muito limitado que requer aplicação de técnicas para prolongar a durabilidade das flores, tal como o emprego de soluções conser- vantes. As soluções mais utilizadas são constituídas por germicidas, fitorreguladores e açúcares (Dias-Tagliacozzo $\&$ Castro, 2002).

Dentre as soluções conservantes, o citrato de 8-hidroxiquinolina (8-HQC) é utilizado no controle de microrganismos das soluções conservantes, ou seja, atua como um excelente redutor do bloqueio dos vasos do xilema na haste. Além disso, interfere no balanço hídrico porque estimula o fluxo de água por meio da seção basal das hastes florais (Mattiuz et al., 2005).

O ácido cítrico, por sua vez, tem como principal função a diminuição do pH das soluções (Mattiuz et al., 2010). Esse efeito do $\mathrm{pH}$ foi explicado por Rogers (1973), ao relatar que soluções ácidas podem inibir a ação de enzimas endógenas, por exemplo, a peroxidase e polifenoloxidase, essenciais para o bloqueio da haste, ou para impedir o desenvolvimento de microrganismos.

As citocininas, tal como a benziladenina, agem como retardadoras da senescência, o que tem sido associado à redução da taxa de perda de proteínas e de RNA, suprimindo a síntese de proteases e prevenindo qualquer aumento na atividade da RNAase (Chitarra \& Chitarra, 2005). Além disso, este fitorregulador é inibidor da degradação da clorofila e estabilizador da respiração.

Os efeitos benéficos dos açúcares, como a sacarose, na senescência das flores de corte são devidos ao fornecimento de substratos para a respiração, manutenção do balanço hídrico que regula a transpiração e aumenta a absorção de água, redução da sensibilidade ao 
etileno e atraso na biossíntese autocatalítica desse gás (Pun \& Ichimura, 2003). Oposto a estas afirmações, Moraes et al. (1999) concluíram que a sacarose de 1 a $2 \%$ na solução de vaso é responsável pela plasmólise e morte do tecido de folhas de rosas de corte.

Considerando a importância da cultivar Vega no mercado nacional (que é uma das mais cultivadas) e a ausência de informações sobre sua conservação pós-colheita, o objetivo foi verificar o efeito do uso de soluções conservantes na fisiologia pós-colheita de rosas vermelhas cortadas cv. Vega, visando à manutenção da qualidade decorativa.

\section{MATERIAL E MÉTODOS}

A cultivar Vega de rosa de corte, adquirida de produtor comercial da cidade de Atibaia-SP, foi transportada até o laboratório da UNESP em Jaboticabal (SP), para realização do experimento. As hastes foram padronizadas em 50 $\mathrm{cm}$, pesadas e submetidas às seguintes soluções de manutenção: 1) Água destilada (controle); 2) 8-HQC (200 mg L-1); 3) 8-HQC (200 mg L-1) + Sacarose (20 $\left.\mathrm{mg} \mathrm{L}{ }^{-1}\right)$; 4) Ácido cítrico (75 $\left.\mathrm{mg} \mathrm{L}^{-1}\right)$; 5) Ácido cítrico $\left(75 \mathrm{mg} \mathrm{L}^{-1}\right)+$ Sacarose (20 mg L-1); 6) 6-benziladenina (60 mg $\left.\mathrm{L}^{-1}\right)$; 7) 6-BA (60 mg L-1) + Sacarose (20 $\left.\mathrm{mg} \mathrm{L}^{-1}\right)$. Essas concentrações foram definidas porque não há relatos científicos recentes das mesmas para avaliar se são ou não adequadas para a espécie com exceção do 8-HQC e da sacarose, que são mais comumente estudados na pós-colheita.

Durante o período experimental, as flores foram mantidas em ambiente com temperatura de $20 \pm 2^{\circ} \mathrm{C}$ e umidade relativa de $67 \pm 3 \%$. Utilizou-se o delineamento experimental inteiramente casualizado, em esquema fatorial composto por sete tratamentos pós-colheita e quatro datas de avaliação $(0,2,4$ e 6 dias). Para cada combinação de fatores foram utilizadas três repetições com três flores cada uma.

Após cada data de avaliação, a massa fresca das flores foi obtida por pesagem. A variação da massa fresca foi calculada em relação à massa inicial.

Para a avaliação da atividade respiratória, cada erlenmeyer, foi colocado em recipiente plástico, com capacidade para $15 \mathrm{~L}$, que era fechado hermeticamente, por 2 horas, nas condições do armazenamento. $\mathrm{O}$ teor de $\mathrm{CO}_{2}$ produzido foi quantificado pelo analisador de gases PBI-Dansensor 9900, ao retirar-se amostras da atmosfera do interior dos recipientes. Para o cálculo da taxa respiratória levou-se em consideração a quantidade de $\mathrm{CO}_{2}$ produzido, o volume do frasco, a massa fresca das flores e o tempo em que o frasco permaneceu fechado.

As avaliações referentes a escurecimento, turgescência e curvatura das hastes foram efetuadas utilizando-se critério de notas: Escurecimento das pétalas (notas de 4 a 1, onde 4= nenhuma pétala escurecida; $3=5$ a 19\%; $2=20$ a $29 \%$ e $1=30 \%$ ou mais de pétalas escurecidas); Turgescência (notas de 4 a 1 , onde 4= túrgida; $3=$ levemente murcha; $2=$ murcha e $1=$ totalmente murcha); Curvatura da flor em relação ao pedúnculo (notas de 4 a 1, onde $4=$ flor reta; $3=$ ângulo entre 1 e $30^{\circ} ; 2=$ ângulo entre 31 e $90^{\circ}$ e $1=$ ângulo maior que $90^{\circ}$, considerado descarte comercial).

O conteúdo relativo de água (CRA) das pétalas foi avaliado coletando-se 10 discos com 10 mm de diâmetro, das pétalas das rosas de cada repetição. Estes discos foram pesados e imersos em água destilada, para hidratação, por 4 horas. Após este período, os discos foram secados superficialmente com papel toalha, colocados em pesa-filtros (recipientes usados para pesagem de materiais sólidos), pesados e levados para estufa a $70^{\circ} \mathrm{C}$, por 12 horas, para secagem e pesagem. $\mathrm{O}$ cálculo do CRA foi feito utilizando-se a equação de Kramer (1983).

Os teores dos carboidratos solúveis foram determinados utilizando-se o método fenol-sulfúrico (Dubois et al., 1956) e os de carboidratos redutores pelo método descrito por Honda et al. (1982).

Para extração e determinação do conteúdo de antocianina, foram retiradas amostras das pétalas das rosas, pesando-se $1 \mathrm{~g}$, segundo método de Francis (1982).

A coloração foi determinada pelo colorímetro MINOLTA CR 400, que utiliza o sistema da CIE 1976 (Minolta Corp,
1994). Em todas as repetições, foram feitas análises destrutivas, removendo-se cinco pétalas, ao acaso, para fazer a leitura.

A longevidade foi avaliada em um lote de flores diferente do delineamento experimental proposto nos experimentos, composto por três repetições e três rosas em cada erlenmeyer para cada tratamento. Fundamentou-se em observações visuais diárias da qualidade decorativa das rosas e no auxílio dos demais parâmetros avaliados. O término da longevidade das flores foi considerado quando as mesmas apresentaram-se com escurecimento, abscisão ou murchamento das pétalas e tombamento das hastes.

A massa fresca, atividade respiratória, avaliação visual (escurecimento, turgescência e curvatura), conteúdo relativo de água, açúcares redutores, açúcares solúveis, antocianina, coloração e longevidade foram submetidos à análise de variância pelo Teste F. As médias foram comparadas utilizando-se o Teste de Tukey a 5\% de probabilidade, exceto para variação de massa fresca e atividade respiratória; neste caso foi aplicado apenas o teste de diferença mínima significativa pelo teste de comparações múltiplas, em que as diferenças entre dois tratamentos, quando maior que a soma de dois desvios padrões, foram consideradas significativas ao nível de 5\% de probabilidade, de acordo com Shamaila et al.(1992).

\section{RESULTADOS E DISCUSSÃO}

A solução de manutenção com 8-HQC propiciou a menor perda de massa fresca das rosas durante os seis dias de armazenamento, seguido pelos tratamentos com ácido cítrico e 8-HQC + sacarose (Figura 1). De acordo com Van Doorn \& Woltering (2008), isto ocorreu em virtude dos compostos químicos diminuírem a transpiração, elevarem o potencial osmótico e fornecerem energia para manter a homeostase celular.

Já as soluções de benziladenina, associadas ou não à sacarose, reduziram de forma acentuada a massa fresca e superaram a perda de massa do tratamento com água destilada. Isso indicou 
Tabela 1. Notas atribuídas ao escurecimento, turgescência e curvatura das hastes de rosa 'Vega' submetidas a sete tratamentos de manutenção e mantidas a $20^{\circ} \mathrm{C}$ e $67 \% \mathrm{UR}$ (notes attributed to the darkening, swelling and curvature of the stems of 'Vega' roses subjected to seven maintenance treatments and maintained at $20^{\circ} \mathrm{C}$ and $67 \%$ RH. Jaboticabal, UNESP, 2009.

\begin{tabular}{|c|c|c|c|c|}
\hline \multirow{2}{*}{ Tratamentos } & \multicolumn{4}{|c|}{ Vida de vaso (dias) } \\
\hline & $\mathbf{0}$ & 2 & 4 & 6 \\
\hline & \multicolumn{4}{|c|}{ Escurecimento } \\
\hline Controle & $4,00 \mathrm{aA}^{\alpha}$ & $3,00 \mathrm{bB}$ & $2,11 \mathrm{cC}$ & $1,11 \mathrm{dD}$ \\
\hline 8-HQC ${ }^{\beta}$ & $4,00 \mathrm{aA}$ & $4,00 \mathrm{aA}$ & $3,89 \mathrm{aA}$ & $2,78 \mathrm{bA}$ \\
\hline 8-HQC + sacarose & $4,00 \mathrm{aA}$ & $4,00 \mathrm{aA}$ & $2,56 \mathrm{bB}$ & $1,56 \mathrm{cC}$ \\
\hline Ácido cítrico & $4,00 \mathrm{aA}$ & $4,00 \mathrm{aA}$ & $3,78 \mathrm{aA}$ & $2,22 \mathrm{bB}$ \\
\hline Ácido cítrico + sacarose & $4,00 \mathrm{aA}$ & $3,78 \mathrm{aA}$ & $2,22 \mathrm{bBC}$ & $1,33 \mathrm{cCD}$ \\
\hline Benziladenina & $4,00 \mathrm{aA}$ & $3,00 \mathrm{bB}$ & $2,11 \mathrm{cC}$ & $1,00 \mathrm{dD}$ \\
\hline \multirow[t]{2}{*}{ Benziladenina + sacarose } & $4,00 \mathrm{aA}$ & $3,00 \mathrm{bB}$ & $2,11 \mathrm{cC}$ & $1,00 \mathrm{dD}$ \\
\hline & \multicolumn{4}{|c|}{ Turgescência } \\
\hline Controle & $4,00 \mathrm{aA}$ & $2,56 \mathrm{bA}$ & $1,78 \mathrm{cB}$ & $1,00 \mathrm{~dB}$ \\
\hline 8-HQC & $4,00 \mathrm{aA}$ & $2,89 \mathrm{aA}$ & $2,89 \mathrm{aA}$ & $2,56 \mathrm{bA}$ \\
\hline 8-HQC + sacarose & $4,00 \mathrm{aA}$ & $2,78 \mathrm{bA}$ & $2,67 \mathrm{bA}$ & $1,22 \mathrm{cB}$ \\
\hline Ácido cítrico & $4,00 \mathrm{aA}$ & $2,89 \mathrm{bA}$ & $2,78 \mathrm{bcA}$ & $2,33 \mathrm{cA}$ \\
\hline Ácido cítrico + sacarose & $4,00 \mathrm{aA}$ & $2,78 \mathrm{bA}$ & $2,67 \mathrm{bA}$ & $1,44 \mathrm{cB}$ \\
\hline Benziladenina & $4,00 \mathrm{aA}$ & $2,56 \mathrm{bA}$ & $1,78 \mathrm{cB}$ & $1,00 \mathrm{~dB}$ \\
\hline \multirow[t]{2}{*}{ Benziladenina + sacarose } & $4,00 \mathrm{aA}$ & $2,56 \mathrm{bA}$ & $1,67 \mathrm{cB}$ & $1,00 \mathrm{~dB}$ \\
\hline & \multicolumn{4}{|c|}{ Curvatura } \\
\hline Controle & $4,00 \mathrm{aA}$ & $3,00 \mathrm{bBC}$ & $2,56 \mathrm{cB}$ & $1,33 \mathrm{dBC}$ \\
\hline 8-HQC & $4,00 \mathrm{aA}$ & $4,00 \mathrm{aA}$ & $3,89 \mathrm{aA}$ & $2,78 \mathrm{bA}$ \\
\hline 8-HQC + sacarose & $4,00 \mathrm{aA}$ & $3,33 \mathrm{bB}$ & $2,89 \mathrm{cB}$ & $1,67 \mathrm{~dB}$ \\
\hline Ácido cítrico & $4,00 \mathrm{aA}$ & $4,00 \mathrm{aA}$ & $3,67 \mathrm{aA}$ & $2,67 \mathrm{bA}$ \\
\hline Ácido cítrico + sacarose & $4,00 \mathrm{aA}$ & $3,22 \mathrm{bB}$ & $2,78 \mathrm{cB}$ & $1,56 \mathrm{~dB}$ \\
\hline Benziladenina & $4,00 \mathrm{aA}$ & $2,67 \mathrm{bC}$ & $1,56 \mathrm{cC}$ & $1,00 \mathrm{dC}$ \\
\hline Benziladenina + sacarose & $4,00 \mathrm{aA}$ & $2,67 \mathrm{bC}$ & $1,56 \mathrm{cC}$ & $1,00 \mathrm{dC}$ \\
\hline
\end{tabular}

$\mathrm{O}$ critério de notas foi descrito em Material e Métodos. ${ }^{\alpha=}$ médias seguidas de pelo menos uma letra comum, minúsculas na linha e maiúsculas na coluna, não diferem entre si pelo Teste de Tukey $(\mathrm{p}>0,05) ;{ }^{\beta}=8$-citrato de hidroxiquinolina (the grades were attibuted according to criteria described in Material and Methods. ${ }^{\alpha}=$ means followed by at least one letter in common, small letters in the line and the same capital letter in the column, do not differ significantly from each other by Tukey test $(\mathrm{p}>0.05) ;{ }^{\beta}=8$-hidroxyquinoline citrate).

a possibilidade da benziladenina, na concentração usada, ter exercido um efeito negativo na qualidade de rosas cortadas da cv. Vega. O uso de $60 \mathrm{mg}$ $\mathrm{L}^{-1}$ foi baseado na pesquisa de Mayak \& Halevy (1974), cuja citocinina manteve a turgescência das pétalas. A explicação mais relevante para o presente experimento é a ação tóxica do regulador vegetal, podendo ter sido excessiva para essa cultivar. Outra hipótese para esses resultados seria a interferência negativa na taxa de proteínas e RNAases, pois Chitarra \& Chitarra (2005) citaram a
8QC + sacarose e ácido cítrico apresentaram as menores taxas respiratórias dentre os tratamentos avaliados. Essa menor taxa respiratória está relacionada com a menor utilização dos carboidratos solúveis e redutores como substrato respiratório, o que permitiu a melhor manutenção da qualidade das rosas, que se apresentaram túrgidas e com pouco esgotamento de suas reservas.

As flores mantidas em solução de 8-HQC e ácido cítrico receberam as melhores notas referentes à evolução das características: escurecimento, turgescência e curvatura de hastes de rosas 'Vega'. (Tabela 1). A superioridade do tratamento com 8-HQC pode ser decorrente da ação inibitória sobre fungos, leveduras e bactérias (Brackmann et al., 2000) Van Doorn \& Perik (1990) já haviam verificado que a concentração de $200 \mathrm{mg} \mathrm{L}^{-1}$ de 8-HQC e baixo $\mathrm{pH}$ impediu o bloqueio vascular (quando o vaso é bloqueado o processo de transpiração continua e não há ganho líquido de água pelo tecido da flor) em quatro cultivares de rosas pela redução do número de bactérias na haste floral.

Quanto ao ácido cítrico, esse também tem ação bactericida e favorece a absorção de água, assim como foi constatado por Nowak \& Rudnicki (1990), mas na concentração de $500 \mathrm{mg} \mathrm{L}^{-1}$. A diferença entre os tratamentos com ácido cítrico e ácido cítrico + sacarose pode ser atribuída à sacarose. A principal função da sacarose é substituir o carboidrato endógeno esgotado pela respiração, retardando o processo de senescência e, consequentemente, preservar o frescor, coloração e longevidade após a colheita. Foi evidenciado que nas soluções associadas com a sacarose ocorreu o inverso, em especial nas duas últimas avaliações, concordando com Han (2009). No entanto, resultados satisfatórios quanto à associação do 8-HQC e sacarose, por exemplo, foram obtidos em rosas (Liao et al., 2000) e crisântemos (Spricigo et al., 2010).

A partir da segunda avaliação, as soluções de benziladenina associada ou não à sacarose não diferiram do controle e apresentaram as menores notas qualitativas (Tabela 1). Foi observada a aceleração da senescência das rosas ao exibirem pétalas enegrecidas, murchas 


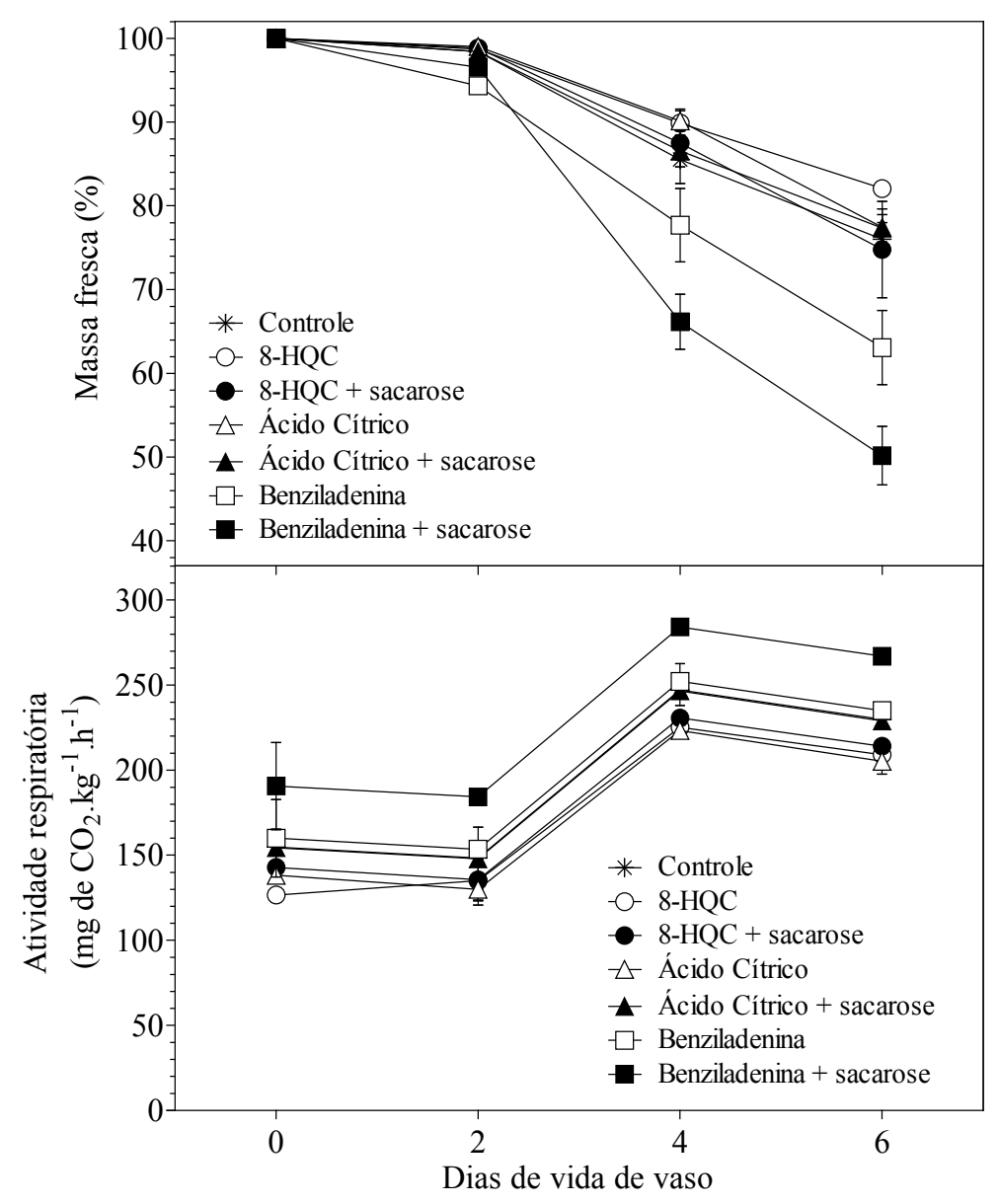

Figura 1. Variação na massa fresca acumulada (\%) e na atividade respiratória (mg de $\mathrm{CO}_{2} /$ $\mathrm{kg} / \mathrm{h}$ ) de hastes de rosas 'Vega', submetidas a sete tratamentos de manutenção e mantidas a $20^{\circ} \mathrm{C}$ e $67 \%$ UR. Barras verticais indicam a média \pm desvio padrão (variation in accumulated fresh weight (\%) and respiratory activity ( $\mathrm{mg}$ of $\left.\mathrm{CO}_{2} / \mathrm{kg} / \mathrm{h}\right)$ of stems of 'Vega' roses, submitted to seven maintenance treatments and kept at $20^{\circ} \mathrm{C}$ and $67 \%$ UR. Data shown are mean \pm standard deviation). Jaboticabal, UNESP, 2009.

Tabela 2. Conteúdo relativo de água (\%) de pétalas de rosa 'Vega' submetidas a sete tratamentos de manutenção e mantidas a $20^{\circ} \mathrm{C}$ e $67 \% \mathrm{UR}$ (relative water contente (\%) of petals of 'Vega' roses subjected to seven maintenance treatments and maintained at $20^{\circ} \mathrm{C}$ and $67 \%$ RH). Jaboticabal, UNESP, 2009.

\begin{tabular}{llllc}
\hline \multirow{2}{*}{ Tratamentos } & \multicolumn{4}{c}{ Vida de vaso (dias) } \\
\cline { 2 - 5 } & \multicolumn{1}{c}{$\mathbf{0}$} & $\mathbf{2}$ & $\mathbf{4}$ & $\mathbf{6}$ \\
\hline Controle & $90,11 \mathrm{aAB}{ }^{\alpha}$ & $86,88 \mathrm{aA}$ & $82,88 \mathrm{aAB}$ & $40,91 \mathrm{bD}$ \\
8-HQC & $98,89 \mathrm{aA}$ & $94,57 \mathrm{abA}$ & $91,44 \mathrm{ab}$ & $86,65 \mathrm{bA}$ \\
8-HQC + sacarose & $88,89 \mathrm{aB}$ & $88,83 \mathrm{aA}$ & $82,65 \mathrm{aAB}$ & $56,89 \mathrm{bC}$ \\
Ácido cítrico & $91,18 \mathrm{aAB}$ & $89,47 \mathrm{aA}$ & $88,24 \mathrm{aAB}$ & $72,04 \mathrm{bB}$ \\
Ácido cítrico + sacarose & $90,24 \mathrm{aAB}$ & $86,96 \mathrm{abA}$ & $81,10 \mathrm{bBC}$ & $57,14 \mathrm{cC}$ \\
Benziladenina & $88,98 \mathrm{aAB}$ & $86,65 \mathrm{aA}$ & $72,20 \mathrm{bC}$ & $43,16 \mathrm{cD}$ \\
Benziladenina + sacarose & $88,63 \mathrm{aB}$ & $85,54 \mathrm{aA}$ & $54,61 \mathrm{bD}$ & $37,05 \mathrm{cD}$ \\
\hline
\end{tabular}

$\alpha=$ médias seguidas de pelo menos uma letra comum, minúsculas nas linhas e maiúsculas na coluna, não diferem entre si pelo Teste de Tukey $(\mathrm{p}>0,05) \cdot{ }^{\beta}=8$-citrato de hidroxiquinolina; $\left({ }^{\alpha}=\right.$ means followed by at least one letter in common, small letter in the line and the same capital letter in the column, do not differ significantly from each other by Tukey test $(p>0,05)$. $\beta=8$-hydroxyquinoline citrate). e com "bent-neck", tornando as hastes inaceitáveis para comercialização. $\mathrm{O}$ tombamento das hastes (bent-neck) manifesta-se pela deposição de compostos orgânicos no lúmen dos vasos xilemáticos ou ainda pela exudação de látex (Finger et al., 2003), pelo crescimento de microrganismos ou pela formação de bolhas de ar.

Foi verificada redução do conteúdo relativo de água em todos os tratamentos, sendo mais expressiva no tratamento com benziladenina associada à sacarose a partir da segunda avaliação (Tabela 2). Os tratamentos com ácido cítrico associado ou não à sacarose e 8-HQC acrescido de sacarose, ocuparam posição intermediária, uma vez que a solução com 8-HQC foi significativamente diferente dos demais, apresentando a menor redução do conteúdo relativo de água no período experimental, o que indica que esse tratamento manteve o balanço hídrico das pétalas de rosa. Isso pode ser atribuído à ação do composto no fechamento dos estômatos, como comprovado por Marousky (1969) em rosas.

Quanto aos níveis de carboidratos solúveis e redutores, esses foram reduzidos ao longo dos dias de vida de vaso. Ao contrário disso, para os tratamentos em que foi adicionada sacarose, ocorreram níveis crescentes dos dois carboidratos (Tabela 3), ocasionados pela sacarose exógena inserida à solução. Isso está em concordancia com os resultados de Kaltaler \& Steponkus (1974) em rosas 'American Beauty' acondicionadas em solução com $2 \%$ de sacarose e $200 \mathrm{mg}$ $\mathrm{L}^{-1}$ de 8-HQC. Mesmo com a adição de sacarose, os tratamentos com benziladenina, ácido cítrico e 8-HQC não demonstraram resultados satisfatórios. Isso pode ser explicado pela maior taxa respiratória (Figura 1) dos mesmos, quando comparado com os outros tratamentos, visto que o esgotamento das reservas de carboidratos, principalmente em função da respiração, ou outros fatores inerentes ao metabolismo vegetal, agravam os sintomas de senescência.

A maior redução dos carboidratos solúveis e redutores foi verificada no tratamento com benziladenina, enquanto que o tratamento com 8-HQC manteve os melhores níveis de carboidratos solú- 
Tabela 3. Conteúdo de carboidratos solúveis e redutores ( $\mathrm{g}$ de glicose/100 g) e conteúdo de antocianinas $(\mathrm{mg} / 100 \mathrm{~g})$ de pétalas de rosa 'Vega' submetidas a sete tratamentos de manutenção e mantidas a $20^{\circ} \mathrm{C}$ e $67 \% \mathrm{UR}$ (content of soluble carbohydrates and reducing sugars ( $\mathrm{g}$ of glucose/100 g) and content of anthocyanins (mg/100 g) of petals of 'Vega' roses subjected to seven maintenance treatments and maintained at $20^{\circ} \mathrm{C}$ and $67 \% \mathrm{RH}$ ). Jaboticabal, UNESP, 2009.

\begin{tabular}{|c|c|c|c|c|}
\hline \multirow{2}{*}{ Tratamentos } & \multicolumn{4}{|c|}{ Vida de vaso (dias) } \\
\hline & $\mathbf{0}$ & 2 & 4 & 6 \\
\hline & \multicolumn{4}{|c|}{ Caboidratos solúveis } \\
\hline Controle & $7,00 \mathrm{aC}^{\alpha}$ & $6,35 \mathrm{bB}$ & $5,17 \mathrm{cD}$ & $4,72 \mathrm{dE}$ \\
\hline $8-\mathrm{HQC}^{\beta}$ & $7,77 \mathrm{aA}$ & $7,62 \mathrm{aA}$ & $6,86 \mathrm{bA}$ & $6,26 \mathrm{cB}$ \\
\hline 8-HQC + sacarose & $4,84 \mathrm{dF}$ & $5,40 \mathrm{cD}$ & $6,58 \mathrm{bA}$ & $8,04 \mathrm{aA}$ \\
\hline Ácido cítrico & $7,41 \mathrm{aB}$ & $6,32 \mathrm{bB}$ & $6,03 \mathrm{cB}$ & $5,25 \mathrm{dD}$ \\
\hline Ácido cítrico + sacarose & $5,60 \mathrm{cD}$ & $5,93 \mathrm{bcC}$ & $6,04 \mathrm{bB}$ & $6,21 \mathrm{aB}$ \\
\hline Benziladenina & $7,03 \mathrm{aC}$ & $6,16 \mathrm{bBC}$ & $5,51 \mathrm{cC}$ & $3,94 \mathrm{dF}$ \\
\hline \multirow[t]{2}{*}{ Benziladenina + sacarose } & $5,26 \mathrm{bE}$ & $5,49 \mathrm{bD}$ & $5,77 \mathrm{aBC}$ & $5,83 \mathrm{aC}$ \\
\hline & \multicolumn{4}{|c|}{ Carboidratos redutores } \\
\hline Controle & $4,19 \mathrm{aC}$ & $4,16 \mathrm{abC}$ & $3,93 \mathrm{bC}$ & $3,25 \mathrm{cF}$ \\
\hline 8-HQC & $6,61 \mathrm{aA}$ & $6,53 \mathrm{abA}$ & $6,38 \mathrm{bA}$ & $6,00 \mathrm{cA}$ \\
\hline 8-HQC + sacarose & $3,28 \mathrm{cD}$ & $3,49 \mathrm{cD}$ & $4,23 \mathrm{bB}$ & $5,33 \mathrm{aB}$ \\
\hline Ácido cítrico & $4,71 \mathrm{aB}$ & $4,63 \mathrm{aB}$ & $4,37 \mathrm{bB}$ & $3,92 \mathrm{cE}$ \\
\hline Ácido cítrico + sacarose & $3,39 \mathrm{cD}$ & $3,55 \mathrm{cD}$ & $4,11 \mathrm{bBC}$ & $4,70 \mathrm{aC}$ \\
\hline Benziladenina & $4,26 \mathrm{aC}$ & $4,17 \mathrm{aC}$ & $3,85 \mathrm{bC}$ & $3,24 \mathrm{cF}$ \\
\hline \multirow[t]{2}{*}{ Benziladenina + sacarose } & $3,13 \mathrm{cD}$ & $3,52 \mathrm{bD}$ & $4,32 \mathrm{aB}$ & $4,41 \mathrm{aD}$ \\
\hline & \multicolumn{4}{|c|}{ Antocianina } \\
\hline Controle & $244,32 \mathrm{bA}$ & $252,13 \mathrm{bA}$ & $263,66 \mathrm{bA}$ & $367,84 \mathrm{aBC}$ \\
\hline 8-HQC & $226,79 \mathrm{aA}$ & $236,18 \mathrm{aA}$ & $244,44 \mathrm{aA}$ & $252,39 \mathrm{aD}$ \\
\hline 8-HQC + sacarose & $216,83 \mathrm{bA}$ & $229,48 \mathrm{bA}$ & $243,42 \mathrm{bA}$ & $280,88 \mathrm{aD}$ \\
\hline Ácido cítrico & $220,73 \mathrm{bA}$ & $229,20 \mathrm{bA}$ & $240,21 \mathrm{abA}$ & $274,34 \mathrm{aD}$ \\
\hline Ácido cítrico + sacarose & $241,27 \mathrm{bA}$ & $244,32 \mathrm{bA}$ & $246,76 \mathrm{bA}$ & $338,32 \mathrm{aC}$ \\
\hline Benziladenina & $251,44 \mathrm{bA}$ & $253,82 \mathrm{bA}$ & $254,84 \mathrm{bA}$ & $381,75 \mathrm{aB}$ \\
\hline Benziladenina + sacarose & $241,27 \mathrm{bA}$ & $244,66 \mathrm{bA}$ & $248,39 \mathrm{bA}$ & $434,01 \mathrm{aA}$ \\
\hline
\end{tabular}

veis e redutores (Tabela 3), disponibilizando maior quantidade de energia para prolongar a vida de vaso das flores. Isso pode ser corroborado com a informação de que as flores de corte com maior concentração de carboidratos apresentam maior durabilidade pós-colheita (Marissen, 2001).

Quanto ao teor de antocianina, na primeira e segunda avaliação, não houve diferença significativa entre as soluções de manutenção (Tabela 3). Isso é devido aos poucos dias decorridos a partir da instalação do experimento, já que inferior de antocianinas em pétalas de rosas, não permitindo o escurecimento das mesmas.

A diminuição dos valores de coloração das pétalas obtidos pela luminosidade, ângulo de cor e cromaticidade foi constante em todos os tratamentos, e a maioria das soluções não mantiveram a cor das pétalas (Tabela 4). As flores tratadas com 8-HQC, ácido cítrico, ácido cítrico+sacarose e 8-HQC+sacarose, respectivamente, apresentaram a menor redução da luminosidade e não apresentaram diferença até o último dia de avaliação. Ao contrário disso, o tratamento com benziladenina associado à sacarose promoveu a maior redução dessa variável, indicando escurecimento das pétalas neste último tratamento. Em relação ao ângulo de cor, houve redução significativa durante os dias de avaliação, sendo maior nas hastes que estavam na solução de benziladenina associada ou não à sacarose. $\mathrm{O}$ tratamento com 8-HQC obteve a melhor estabilidade da cor vermelha, embora tenha apresentado decréscimos nos resultados obtidos para a cromaticidade durante o período avaliado, evidenciando a melhor manutenção dos pigmentos. Isso concorda com Marousky \& Carlyle (1985), que observaram que o uso do 8-HQC em hastes cortadas de rosa 'Better Times' preservou a cor característica da flor ao longo do armazenamento.

O tratamento com 8-HQC possibilitou a maior longevidade das rosas cv. Vega (dez dias de vida de vaso) e diferiu ao nível de $5 \%$ de significância das demais soluções. As outras soluções conservantes também tiveram diferenças significativas entre si, com exceção do ácido cítrico+sacarose e controle (sete dias de vida de vaso) e também da benziladenina e benziladenina + sacarose que se igualaram a seis dias de vida de vaso.

A durabilidade das rosas tratadas com 8-HQC promoveu o melhor desempenho das hastes ao referido composto químico em todos os atributos avaliados nesta pesquisa, embora nem sempre tenha diferido dos demais, principalmente do ácido cítrico. Não obstante, os tratamentos com benziladenina não corresponderam à qualidade decorativa das flores, sendo considerados, em al- 
Tabela 4. Coloração das pétalas de rosa 'Vega', submetidas a sete tratamentos de manutenção e mantidas a $20^{\circ} \mathrm{C}$ e $67 \%$ UR (color of petals of 'Vega' roses subjected to seven maintenance treatments and maintained at $20^{\circ} \mathrm{C}$ and $67 \%$ RH). Jaboticabal, UNESP, 2009.

\begin{tabular}{|c|c|c|c|c|}
\hline \multirow{2}{*}{ Tratamentos } & \multicolumn{4}{|c|}{ Vida de vaso (dias) } \\
\hline & $\mathbf{0}$ & 2 & 4 & 6 \\
\hline & \multicolumn{4}{|c|}{ Luminosidade } \\
\hline Controle & $38,91 \mathrm{aA}^{\alpha}$ & $38,40 \mathrm{aA}$ & $38,01 \mathrm{aA}$ & $35,36 \mathrm{bBC}$ \\
\hline $8-\mathrm{HQC}^{\beta}$ & $39,17 \mathrm{aA}$ & $38,95 \mathrm{abA}$ & $38,44 \mathrm{abA}$ & $37,54 \mathrm{bA}$ \\
\hline 8-HQC + sacarose & $39,31 \mathrm{aA}$ & $38,29 \mathrm{abA}$ & $37,78 \mathrm{bA}$ & $36,36 \mathrm{cAB}$ \\
\hline Ácido cítrico & $38,96 \mathrm{aA}$ & $38,68 \mathrm{aA}$ & $37,56 \mathrm{abAB}$ & $36,91 \mathrm{bAB}$ \\
\hline Ácido cítrico + sacarose & $38,87 \mathrm{aA}$ & $38,41 \mathrm{aA}$ & $37,93 \mathrm{aA}$ & $36,23 \mathrm{bAB}$ \\
\hline Benziladenina & $37,90 \mathrm{aAB}$ & $37,41 \mathrm{abA}$ & $36,01 \mathrm{bBC}$ & $34,48 \mathrm{cCD}$ \\
\hline \multirow[t]{2}{*}{ Benziladenina + sacarose } & $36,82 \mathrm{aB}$ & $35,19 \mathrm{bB}$ & $34,58 \mathrm{bC}$ & $33,00 \mathrm{cD}$ \\
\hline & \multicolumn{4}{|c|}{ Ângulo de cor } \\
\hline Controle & $28,11 \mathrm{aA}$ & $27,32 \mathrm{abAB}$ & $26,42 \mathrm{bBC}$ & $24,52 \mathrm{cBC}$ \\
\hline 8-HQC & $28,58 \mathrm{aA}$ & $28,44 \mathrm{aA}$ & $28,13 \mathrm{aAB}$ & $27,44 \mathrm{bA}$ \\
\hline 8-HQC + sacarose & $28,09 \mathrm{aA}$ & $27,83 \mathrm{abAB}$ & $26,92 \mathrm{bBC}$ & $24,96 \mathrm{cB}$ \\
\hline Ácido cítrico & $28,11 \mathrm{aA}$ & $27,79 \mathrm{aAB}$ & $27,27 \mathrm{aAB}$ & $25,43 \mathrm{bB}$ \\
\hline Ácido cítrico + sacarose & $28,29 \mathrm{aA}$ & $27,56 \mathrm{abAB}$ & $26,65 \mathrm{bBC}$ & $25,03 \mathrm{cB}$ \\
\hline Benziladenina & $27,69 \mathrm{aA}$ & $27,45 \mathrm{aAB}$ & $25,94 \mathrm{bC}$ & $23,82 \mathrm{cC}$ \\
\hline \multirow[t]{2}{*}{ Benziladenina + sacarose } & $27,64 \mathrm{aA}$ & $27,29 \mathrm{aB}$ & $26,17 \mathrm{bBC}$ & $23,62 \mathrm{cC}$ \\
\hline & \multicolumn{4}{|c|}{ Cromaticidade } \\
\hline Controle & $64,87 \mathrm{aAB}$ & $64,60 \mathrm{aA}$ & $62,92 \mathrm{bBC}$ & $61,01 \mathrm{cC}$ \\
\hline 8-HQC & $65,79 \mathrm{aA}$ & $65,42 \mathrm{abA}$ & $64,93 \mathrm{abA}$ & $64,22 \mathrm{bA}$ \\
\hline 8-HQC + sacarose & $65,30 \mathrm{aA}$ & $64,99 \mathrm{abA}$ & $63,87 \mathrm{bAB}$ & $62,03 \mathrm{cBC}$ \\
\hline Ácido cítrico & $65,82 \mathrm{aA}$ & $65,36 \mathrm{aA}$ & $64,95 \mathrm{aA}$ & $63,24 \mathrm{bAB}$ \\
\hline Ácido cítrico + sacarose & $65,03 \mathrm{aAB}$ & $64,62 \mathrm{abA}$ & $63,38 \mathrm{bAB}$ & $61,35 \mathrm{cC}$ \\
\hline Benziladenina & $64,48 \mathrm{aAB}$ & $64,20 \mathrm{abAB}$ & $62,96 \mathrm{bBC}$ & $60,56 \mathrm{cCD}$ \\
\hline Benziladenina + sacarose & $63,53 \mathrm{aB}$ & $62,89 \mathrm{abB}$ & $61,75 \mathrm{bC}$ & $59,38 \mathrm{cD}$ \\
\hline
\end{tabular}

guns parâmetros, inferiores ao controle.

\section{AGRADECIMENTOS}

À Fundação de Amparo à Pesquisa do Estado de São Paulo (FAPESP) pela concessão de bolsa de estudos (Proc. n. 07/57918-9).

\section{REFERÊNCIAS}

BARGUIL BM; MEIRELES B; VIANA, FMP; MOSCA JL. 2010. Características morfológicas e fitossanitárias de variedades de roseira na etapa de classificação. Ciência Rural. 40: 1545-1549.

BRACKMANN A; BELLÉ RA; VIZZOTTO M; LUNARDI R. 2000. Armazenamento de crisântemos Dedranthema grandiflora cv. Red refocus em diferentes temperaturas e soluções conservantes. Revista Brasileira de Agrociência 6: 19-23.

CHITARRA MIF; CHITARRA AB. 2005. Póscolheita de frutos e hortaliças: fisiologia e manuseio. Lavras: ESAL. 785p.

DIAS-TAGLIACOZZO GM; CASTRO CEF. 2002. Fisiologia da pós-colheita de espécies ornamentais. In: WACHOWICZ CM.; CARVALHO RIN (eds). Fisiologia vegetal: produção e pós-colheita. Curitiba: Champagnat. p. 359-382.

DUBOIS M; GILLES KA; HAMILTON JK; REBERS PA; SMITH F. 1956. Colorimetric method for determination of sugars and related substances. Analytical Chemistry 28: 350-356.

FINGER FL; BARBOSA JG; GROSSI, JAS; MORAES PJD. 2003. Colheita, classificação e armazenamento de inflorescências de crisântemos. In: BARBOSA JG. Crisântemos. Viçosa: Aprenda Fácil. p.123-140.

FRANCIS FJ. 1982. Analysis of anthocyanins. In: MARKAKIS P. Anthocyanins as food colors. New York: Academic Press. p. 182-205.

HAN SS. 2009. 15 jan. Sugar and acidity in preservative solutions for field-grown cut flowers. Disponível em http://www.umass.edu HONDA S; NISHIMURA Y; TAKAHASHI M; CHIBA H; KAKEHI KA. 1982. Manual method for spectrophotometric determination of reducing carbohydrates with 2-cyanoacetamide. Analytical Biochemistry 119: 194-199.

IBRAFLOR - INSTITUTO BRASILEIRO DE FLORICULTURA. 2011, 04 de agosto. Uma visão do mercado de flores. Disponível em http://www.ibraflor.com

JUNQUEIRAAH; PEETZ MS. 2007. Inteligência comercial no mercado de flores. In: Semana internacional da fruticultura, floricultura e agroindústria, 14. Resumos... Fortaleza: Instituto Frutal (CD-Rom).

KALTALER REL; STEPONKUS PL. 1974. Uptake and metabolism of sucrose in cut roses. Journal of the American Society for Horticultural Science 99: 490-493.

KRAMER PJ. 1983. Water relations of plants. New York: Academic Press. 489 p.

LIAO LJ; LIN YH; HUANG K-L; CHEN WS; 
CHENG Y-M. 2000. Postharvest life of cut rose flowers as affected by silver thiosulphate and sucrose. Botanical Bulletin of Academic Sinica 41: 299-303.

MARISSEN N. 2001. Effects of pre-harvest light intensity and temperature on carbohydrate levels and vase life of cut roses. Acta Horticulturae 543: 331-343.

MAROUSKY FJ. 1969. Vascular blockage, water absorption, stomatal opening, and respiration of cut "Better Times" roses treated with 8-hydroxyquinoline citrate and sucrose. Journal of the American of Society for Horticultural Science 94: 223-226.

MAROUSKY FJ; CARLYLE TC. 1985. Postharvest color changes in red rose petals. Proceedings of the Florida State Horticultural Society 98: 137-139.

MATTIUZ CFM; RODRIGUES TJD; MATTIUZ BH; PIVETTA KFL. 2005. Water relations of cut inflorescences of Alpinia purpurata treated with seven pulsing solutions. Acta Horticulturae 683: 363-368.

MATTIUZ CFM; RODRIGUES TJD; MATTIUZ BH; PIETRO J; MARTINS RN. 2010.
Armazenamento refrigerado de inflorescências cortadas de Oncidium varisosum 'Samurai'. Ciência Rural 40: 2288-2293.

MAYAK S.; HALEVY H. 1974. The action of kinetin in improving the water balance and delaying senescence processes of cut rose flowers. Physiologia Plantarum 32: 330-336.

MINOLTA CORP. 1994. Precise color communication: color control from feeling to instrumentation. Ramsey: Minolta Corporation Instrument System Division. 49p.

MORAES PJ; CECON PR; FINGER FL; BARBOSA JG; ALVARES VS. 1999. Efeito da refrigeração e do condicionamento em sacarose sobre a longevidade de inflorescências de Strelitzia reginae AIT. Revista Brasileira de Horticultura Ornamental 5: 151-156.

NOWAK J; RUDNICKI RM. 1990. Postharvest handling and storage of cut flowers, florist greens and potted plants. Portland: Timber Press, 210p.

PUN UK.; ICHIMURA K. 2003. Role of sugars in senescence and biosynthesis of ethylene in cut flowers. Japan Agricultural Research Quarterly 37: 219-224.
ROGERS MN. 1973. An historical and critical review of postharvest physiology research on cut flowers. HortScience 8: 189-194.

SHAMAILA M; POWRIE WD; SKURA B. 1992. Sensory evaluation of strawberry fruit stored under modified atmosphere packaging (MAP) by quantitative descriptive analysis. Journal of Food Science 57: 1168-1172.

SPRICIGO PC.; MATTIUZ BH.; PIETRO J.; MATTIUZ CFM.; OLIVEIRA MEM. 2010. Uso de soluções de manutenção, associadas ou não a sacarose, na pós-colheita de hastes de Chrysanthemum morifolium cv. Dragon. Ciência e Agrotecnologia, 34:1238-1244.

TAKANE RJ; TADEU P; CASARINI E. 2007. Cultivo de rosas. Brasília: LK. $171 \mathrm{p}$.

VAN DOORN WG; WOLTERING EJ. 2008. Physiology and molecular biology of petal senescence. Journal of Experimental Botany 59: 453-480.

VAN DOORN WG; PERIK RR. 1990. Hydroxyquinoline citrate and low $\mathrm{pH}$ prevent vascular blockage in stem of cut rose flowers by reducing the number of bacteria. Journal of the American of Society for Horticultural Science 115: 979-981. 